\title{
Loss of astrocyte polarity marks blood-brain barrier impairment during experimental autoimmune encephalomyelitis
}

\author{
Karen Wolburg-Buchholz • Andreas F. Mack • \\ Esther Steiner · Friederike Pfeiffer • \\ Britta Engelhardt · Hartwig Wolburg
}

Received: 20 February 2009/Revised: 14 May 2009/ Accepted: 7 June 2009/Published online: 17 June 2009

(c) Springer-Verlag 2009

\begin{abstract}
In multiple sclerosis (MS), and its animal model experimental autoimmune encephalomyelitis (EAE), dysfunction of the blood-brain barrier (BBB) leads to edema formation within the central nervous system. The molecular mechanisms of edema formation in EAE/MS are poorly understood. We hypothesized that edema formation is due to imbalanced water transport across the BBB caused by a disturbed crosstalk between BBB endothelium and astrocytes. Here, we demonstrate at the light microscopic and ultrastructural level, the loss of polarized localization of the water channel protein aquaporin-4 (AQP4) in astrocytic endfeet surrounding microvessels during EAE. AQP4 was found to be redistributed over the entire astrocytic cell surface and lost its arrangement in orthogonal arrays of intramembranous particles as seen in the freeze-fracture replica. In addition, immunostaining for the astrocytic extracellular matrix receptor $\beta$-dystroglycan disappeared from astroglial membranes in the vicinity of inflammatory cuffs, whereas immunostaining for the dystroglycan ligands agrin and laminin in the perivascular basement membrane remained unchanged. Our data suggest that during EAE, loss of $\beta$-dystroglycan-mediated astrocyte foot process anchoring to the basement
\end{abstract}

K. Wolburg-Buchholz $\cdot$ H. Wolburg ( $\square)$

Institute of Pathology, University of Tübingen,

Liebermeisterstraße 8, 72076 Tübingen, Germany

e-mail: hartwig.wolburg@med.uni-tuebingen.de

\section{A. F. Mack}

Institute of Anatomy, University of Tübingen,

72076 Tübingen, Germany

E. Steiner · F. Pfeiffer - B. Engelhardt

Theodor Kocher Institute, University of Bern,

3012 Bern, Switzerland membrane leads to loss of polarized AQP4 localization in astrocytic endfeet, and thus to edema formation in EAE.

Keywords Agrin - Aquaporin-4 - Astrocytes ·

Blood-brain barrier $\cdot$ EAE $\cdot$ Inflammation

\section{Introduction}

Homeostasis of the central nervous system (CNS) microenvironment is essential for its normal function and is maintained by the blood-brain barrier (BBB). During pathological conditions of the CNS such as in multiple sclerosis (MS), or in its animal model experimental autoimmune encephalomyelitis (EAE), loss of barrier properties of the highly specialized cerebral endothelium leads to edema formation and exacerbation of clinical disease $[24,30]$. The BBB is formed by highly specialized endothelial cells, which inhibit transcellular passage of molecules across the barrier by an extremely low pinocytotic activity and restrict the paracellular diffusion of hydrophilic molecules due to an elaborate network of complex tight junctions (TJ) between the endothelial cells (as reviewed in $[9,37])$. While the endothelial cells form the barrier proper, integrity of the BBB critically depends on the continuous crosstalk between the cellular elements within the neurovascular unit. Pericytes embedded into the endothelial basement membrane, are required for the maturation of the BBB [12]. In addition, astrocytes closely embrace CNS microvessels covering almost entirely the abluminal vascular surface with their endfeet. Astrocytes deposit a second basal lamina around CNS microvessels, referred to as glial basal lamina or glial limiting membrane. The endothelial and the glial basement membranes differ in their laminin composition with laminins 411 and 511 in the 
endothelial basal lamina, versus laminins 111 and 211 deposited in the glial limiting membrane [1, 29].

In EAE, inflammatory cells therefore have to breach the endothelial barrier and both basal laminae before reaching the CNS parenchyma proper. Activity of matrix metalloproteinases MMP2 and MMP9 is required for leukocyte migration across the astroglial basal lamina but dispensable for leukocyte entry across the endothelial $\mathrm{BBB}$ and the endothelial basal lamina [1]. MMP2 and MMP9 cleave the extracellular matrix receptor $\beta$-dystroglycan localized in the astrocytic endfoot membrane during their passage into the CNS. Besides laminin, $\beta$-dystroglycan binds agrin, a heparan sulfate proteoglycan localized in the basal laminae of the CNS microvessels and proposed to be involved in the development of the BBB [4]. Linkage of astrocytic foot processes to agrin via $\beta$-dystroglycan may therefore be critical for correct astrocyte anchoring in the neurovascular unit (NVU).

Freeze-fracture electron microscopy has revealed astrocyte polarity within the NVU by revealing structural heterogeneity of specific membrane domains on the astroglial surface. Membranes of astrocyte endfeet, but not parenchymal membranes of the astrocyte, are studded with numerous orthogonal arrays of intramembranous particles (OAPs) (as summarized in [36]). The molecular entity found in OAPs is the water channel protein aquaporin-4 (AQP4) [22, 23], suggesting that in EAE loss of astrocyte polarity may lead to loss of directed water transport across astrocytes causing vascular or cytotoxic edema.

In the past, an altered expression of AQP4 in inflammatory diseases of the brain has been described. AokiYoshida et al. [3] and Sinclair et al. [28] found an enhanced expression of AQP4 in human multiple sclerosis. In contrast, in the autoimmune disorder neuromyelitis optica (NMO), in which the autoantigen is AQP4 [10], AQP4 was found to be absent [28].

In this study, we have investigated the distribution of AQP4 in EAE plaques and demonstrate that in fact edema formation in EAE is accompanied by the loss of polarized localization of AQP4 and the $\mathrm{K}^{+}$channel Kir4.1 on astrocytic foot processes within the NVU. Freeze-fracture analysis confirmed the loss of astrocyte polarity by demonstrating a rearranged architecture of parenchymal membrane domains of astrocytes adjacent to inflammatory cuffs. Failure to maintain cell polarity seemed to be caused by the cleavage of the extracellular matrix receptor $\beta$ dystroglycan at the astrocytic foot process leading to the loss of contact with the basement membrane ligands laminin and agrin, presence of which remains unchanged within the glial limiting membrane. Our data suggest that during EAE the distribution of AQP4 and OAPs in the astrocyte membrane has been changed. It is tempting to speculate that these changes are linked to the loss of $\beta$-dystroglycan, leading to a reduction of astrocyte polarization, and thus to edema formation in EAE.

\section{Materials and methods}

Animals and induction of EAE

Female SJL/N mice were obtained from Harlan (The Netherlands) and used for experiments at the age of 10 weeks. Active EAE (aEAE) was induced by immunization with PLP-peptide as described in detail before [32]. Animals were checked daily and clinical severity (clinical score) was documented as follows: $\varnothing$ without symptoms; $0.5=\operatorname{limp}$ tail; $1=$ weak hind limbs; $2=$ paraplegic; 3 = paraplegic, incontinent and weakness. Animal experiments were performed in accordance to the Swiss legislation with permission of the local authorities.

\section{Antibodies}

The following antibodies were used to detect specific water channel and tight junction proteins: polyclonal rabbit antiaquaporin-4 antiserum (Chemicon, Hofheim, Germany), polyclonal rabbit anti-claudin-3 (Zymed, Invitrogen, Karlsruhe, Germany), polyclonal rabbit anti-claudin-5 [11], polyclonal rabbit anti-occludin (Zymed, Invitrogen, Karlsruhe, Germany), polyclonal rabbit anti-GFAP (1:500; DAKO, Hamburg, Germany), polyclonal rabbit anti-ZO-1 and monoclonal mouse anti-ZO-1 (Zymed, Invitrogen, Karlsruhe, Germany), polyclonal rabbit anti-Kir4.1 (Alomone Labs, Jerusalem, Israel), sheep anti-human fibronectin, FITC-conjugated (1:100; BioTrend, Cologne, Germany), monoclonal mouse anti- $\beta$-dystroglycan (NovoCastra, Newcastle, UK), polyclonal rabbit anti-agrin (provided by Dr. S. Kröger), polyclonal rabbit anti-laminin (DAKO, Hamburg, Germany), monoclonal anti-rat CD45 (BD Pharmingen). If not otherwise mentioned, all antisera were used in a dilution of 1:100.

The secondary goat-anti-mouse, or goat-anti-rabbit antibodies labeled with cyanin-derivative dye $\mathrm{Cy} 2, \mathrm{Cy} 3$ or Cy5 were purchased from Dianova (Hamburg, Germany). For controls, the primary antibody was omitted.

Gel electrophoresis and western blot

Mice with EAE and healthy controls were anesthetized and perfused with cold phosphate buffered saline (PBS). The cerebellum was removed and homogenized in 10 volumes (w/v) of ice-cold RIPA buffer $(50 \mathrm{mM}$ Tris $\mathrm{pH}$ 7.5, $150 \mathrm{mM} \mathrm{NaCl}, 0.5 \%$ Nadeoxycholate, $0.1 \%$ SDS, $1 \%$ NP-40) containing protease inhibitors (Complete Mini 
EDTA-free protease inhibitor cocktail tablets, Roche). After a 5 min centrifugation step at $3,000 \times g$ the supernatant was harvested and the protein concentration was determined with the BCA protein assay kit (Pierce). The samples were mixed with $2 \times$ sample buffer (Laemmli Buffer, $200 \mathrm{mM}$ DTT), $90 \mu \mathrm{g}$ of proteins per sample separated on a $10 \%$ polyacrylamide gel and blotted on nitrocellulose (Whatman Protran transfer membrane, $0.2 \mathrm{mM}$ pore size). After blocking the membrane in PBS containing $2 \%(\mathrm{w} / \mathrm{v})$ BSA (Albumin fraction V, Applichem) for $20 \mathrm{~min}$ at RT, the membrane was incubated overnight with the mouse monoclonal anti $\beta$-actin antibody (A5316, Sigma) diluted 1:1,000 in PBS/2\% BSA at $4{ }^{\circ} \mathrm{C}$. After washing with PBS, the primary antibody was detected by goat-anti-mouse IRDye 680 (LI-COR Biosciences). The rabbit polyclonal anti-AQP4 antibody (AB2218, Millipore) was diluted 1:500 in PBS/2\% BSA and the membrane was incubated for $1 \mathrm{~h}$ at RT. The primary antibody was revealed with goat-anti-rabbit IRDye 800CW (LI-COR Biosciences) and the membrane was scanned with the Odyssey Infrared imaging system. The integrated intensity of the bands was determined using Odyssey 2.1 software. The AQP4 bands were normalized to the corresponding $\beta$-actin bands for each individual sample.

\section{Immunohistochemistry}

Mice with EAE or healthy littermates were killed, and their brains were removed, embedded in Tissue Tek (OCT; Miles, Vogel, Giessen, Germany) and snap-frozen in a 2-methylbutane (Merck, Darmstadt, Germany) bath at $-80^{\circ} \mathrm{C}$. Cryostat sections $(8-12 \mu \mathrm{m}$ thick) were cut using a Reichert-Jung Frigocut 2800 E cryotome (Reichert, Vienna, Austria), mounted on glass slides coated with poly-L-lysine (Sigma), and air dried. To avoid unspecific staining, the sections were blocked by incubation with $5 \%(\mathrm{w} / \mathrm{v})$ skimmed milk, $0.3 \%(\mathrm{w} / \mathrm{v})$ Triton X-100 (Serva, Heidelberg, Germany) and 0.4\% (w/v) $\mathrm{NaN}_{3}$ in TBS for $30 \mathrm{~min}$. Primary antibodies diluted in the same solution were applied overnight at $4{ }^{\circ} \mathrm{C}$. After three washes in TBS for $10 \mathrm{~min}$, sections were incubated for $45 \mathrm{~min}$ with the secondary antibody at room temperature. Following washes in TBS, sections were mounted in Mowiol. Sections were analyzed with a confocal laser scanning microscope (LSM510 META with an Axioplan 2 microscope stand, Zeiss, Göttingen/ Jena, Germany) using lasers at 488, 546, and $633 \mathrm{~nm}$ for excitation with appropriate filter sets. The system's multitrack function was used to generate images for each stain and excitation sequentially. Images were processed using Adobe Photoshop (version 7.0, Adobe, Mountain View, USA).
Immunogold labeling

Post-embedding immunogold labeling was performed on ultrathin sections of Lowicryl-embedded specimens. The tissues were fixed with $3 \%$ paraformaldehyde plus $0.1 \%$ glutaraldehyde in phosphate buffer $\mathrm{pH} 7.2$ for $4 \mathrm{~h}$, then dehydrated and embedded in Lowicryl K4M at low temperature in a Balzers low temperature embedding apparatus (LTE 020; Balzers, Liechtenstein), followed by UV polymerization at low temperature in a Balzers freezer (TTP 010; Balzers, Liechtenstein). The sections were cut on a Leica Ultracut $\mathrm{R}$ at 50-70 nm and mounted on Pioloformcoated nickel grids (SCI, Munich, Germany). For on-section labeling, grids were floated on drops of the following solutions and transferred using a nickel-coated copper loop. Initially, the grids were floated for $5 \mathrm{~min}$ on TBS followed by blocking buffer [5\% (w/v) bovine serum albumin, $0.1 \%$ (v/v) cold water fish skin gelatin (Biotrend), 5\% (v/v) normal goat serum (Biotrend), and $0.04 \% \mathrm{NaN}_{3}$ in TBS] for $30 \mathrm{~min}$ to minimize unspecific binding. The primary antibody was diluted (1:20 or 1:50) in incubation buffer [0.8\% (w/v) bovine serum albumin, $0.1 \%(\mathrm{v} / \mathrm{v})$ cold water fish skin gelatine, and $0.04 \% \mathrm{NaN}_{3}$ in TBS], and the sections were incubated overnight at $4^{\circ} \mathrm{C}$. The sections were washed several times in incubation buffer and subsequently incubated for $1 \mathrm{~h}$ with gold-conjugated secondary antibodies (British Biocell Int., Cardiff, UK) diluted 1:50 in incubation buffer. Following several washes in the same buffer and in TBS, the sections were postfixed with $2 \%$ glutaraldehyde and finally washed in double-distilled water. The sections were contrasted with $8 \%$ watery uranyl acetate. The specimens were observed and documented with a Zeiss EM10A electron microscope (Zeiss, Oberkochen, Germany).

\section{Conventional electron microscopy of vibratome sections}

Mice were transcardially perfused with $2.5 \%$ glutaraldehyde (Paesel-Lorei, Frankfurt, Germany) buffered in $0.1 \mathrm{M}$ cacodylate buffer ( $\mathrm{pH}$ 7.4). Thereafter, the cerebellar and spinal cord tissue was dissected out and postfixed in the identical fixative for additional $4 \mathrm{~h}$, and then stored in cacodylate until further processed. The tissues were sectioned with an Oxford Vibratome (Oxford, UK) into 50$\mu \mathrm{m}$-thick sections, which were consecutively used for embedding and freeze-fracturing (see there). For embedding, the vibratome sections $(1,3,5, \ldots)$ were postfixed in $1 \% \mathrm{OsO}_{4}$ in $0.1 \mathrm{M}$ cacodylate buffer and then dehydrated in an ethanol series $(50,70,96,100 \%)$. The $70 \%$ ethanol was saturated with uranyl acetate for contrast enhancement. Dehydration was completed in propylene oxide. The specimens were embedded in Araldite (Serva, Heidelberg, 
Germany). Semi- and ultra-thin sections were produced on a FCR Reichert Ultracut ultramicrotome (Leica, Bensheim, Germany). The semi-thin sections were used for inspection of inflammatory cuffs. If they were present, conventional electron microcopy was made from this site, and the ultrathin sections were mounted on Pioloform-coated copper grids, contrasted with lead citrate and analyzed and documented with an EM10A electron microscope (Carl Zeiss, Oberkochen, Germany). In this case, the next vibratome sections $(2,4,6, \ldots)$ were used for freeze-fracturing transmission electron microscopy.

\section{Freeze-fracturing}

The vibratome sections produced as described above were cryoprotected for freeze-fracturing in $30 \%$ glycerol and quick-frozen in nitrogen-slush $\left(-210^{\circ} \mathrm{C}\right)$. Subsequently, the specimens were fractured in a Balzer's freeze-fracture device (BAF400D; Balzers, Liechtenstein) at $5 \times 10^{-6}$ mbar and $-150^{\circ} \mathrm{C}$. The fracture faces were shadowed with platinum/ carbon $\left(2 \mathrm{~nm}, 45^{\circ}\right)$ for contrast and carbon $\left(20 \mathrm{~nm}, 90^{\circ}\right)$ for stabilization of the replica. After removing the cell material in $12 \%$ sodium hypochlorite, the replicas were cleaned several times in double-distilled water and mounted on Pioloformcoated copper grids. The replicas were observed using an EM10A electron microscope (Carl Zeiss, Oberkochen, Germany).

\section{Results}

In this study, we investigated inflammatory cuffs in the cerebellum and the spinal cord of mice with different clinical severity of EAE by means of both confocal laser scanning immunohistochemistry and immunogold labeling as well as by means of freeze-fracturing. We used antibodies against CD45 in order to identify inflammatory cells, against endothelial tight junctions (ZO-1, occludin, claudin-3 and claudin-5) in order to test the conditions of the BBB in the postcapillary venules, and against astroglial antigens such as the water channel protein aquaporin-4 (AQP4), the $\mathrm{K}^{+}$channel Kir4.1, glial fibrillary acidic protein (GFAP), and the dystrophin-dystroglycan complex (DDC) ( $\beta$-dystroglycan). In addition, we focused our interest on the extracellular matrix compounds fibronectin, laminin and agrin.

Fibronectin staining indicates leakage of the BBB

Fibronectin immunoreactivity (IR) in non-inflamed or weakly inflamed vessels was restricted to the basal lamina (Fig. 1a). However, where CD45-positive cells had transmigrated the vessel wall lying within the perivascular space, fibronectin formed a meshwork surrounding the inflammatory cells (Fig. 1b). Extended "clouds" of antifibronectin immunoreactivity, were observed around blood vessels in the CNS of mice with more severe EAE (score 3, Fig. 1c). The incongruency of GFAP and fibronectin staining supported the view that fibronectin was not produced by astrocytes, but has rather leaked from the bloodstream across a compromised BBB into the neuropil. Thus, by following the concept of Nag [15, 39], fibronectin staining was used here as an indicator of the barrier characteristics of the BBB rather than a vascular marker.

Tight junction proteins with exception to claudin-3 remain present in inflamed CNS microvessels

The anti-ZO-1 antibody was frequently used in this study to identify tight junctions in endothelial cells. In all conditions tested, ZO-1 staining was not decreased. Similarly, immunoreactivities for occludin and claudin-5 were either scarcely affected or not affected (data not shown). In contrast, anti-claudin-3 immunoreactivity was lost from microvessels surrounded by inflammatory cuffs confirming our previous findings (data not shown [38]). Taken together, the disappearance of claudin-3 immunoreactivity from the junctions of inflamed vessels seems to be a characteristic feature of EAE-related inflammation.

Laminin staining remains stable in endothelial and glial basal laminae

Pan-laminin staining was the most suitable tool to recognize CNS blood vessels. Capillaries as well as postcapillary venules, with or without inflammatory cuffs, stained positive for laminin (Fig. 2b). Intensity of laminin immunofluorescence staining of both the endothelial basal lamina and the glial limiting membrane were maintained irrespective of the degree of inflammation or number of extravasated CD45-positive cells suggesting that laminin was stable and not a target of enzymatic degradation during diapedesis. Figure $6 \mathrm{~d}$ shows an immunogold staining of EAE material demonstrating anti-laminin labeling of glial and endothelial basal laminae.

$\beta$-dystroglycan is lost in astrocytes adjacent to perivascular inflammatory cuffs

$\beta$-dystroglycan immunoreactivity in uninflamed CNS microvessels is regularly detected in the perivascular astroglial endfoot membranes (Fig. 2a). By immunogold labeling using a monoclonal antibody against $\beta$-dystroglycan, we found gold grains exclusively in astrocytic, not endothelial membranes of perivascular astrocytic endfoot membranes (data not shown). Confirming the study of 

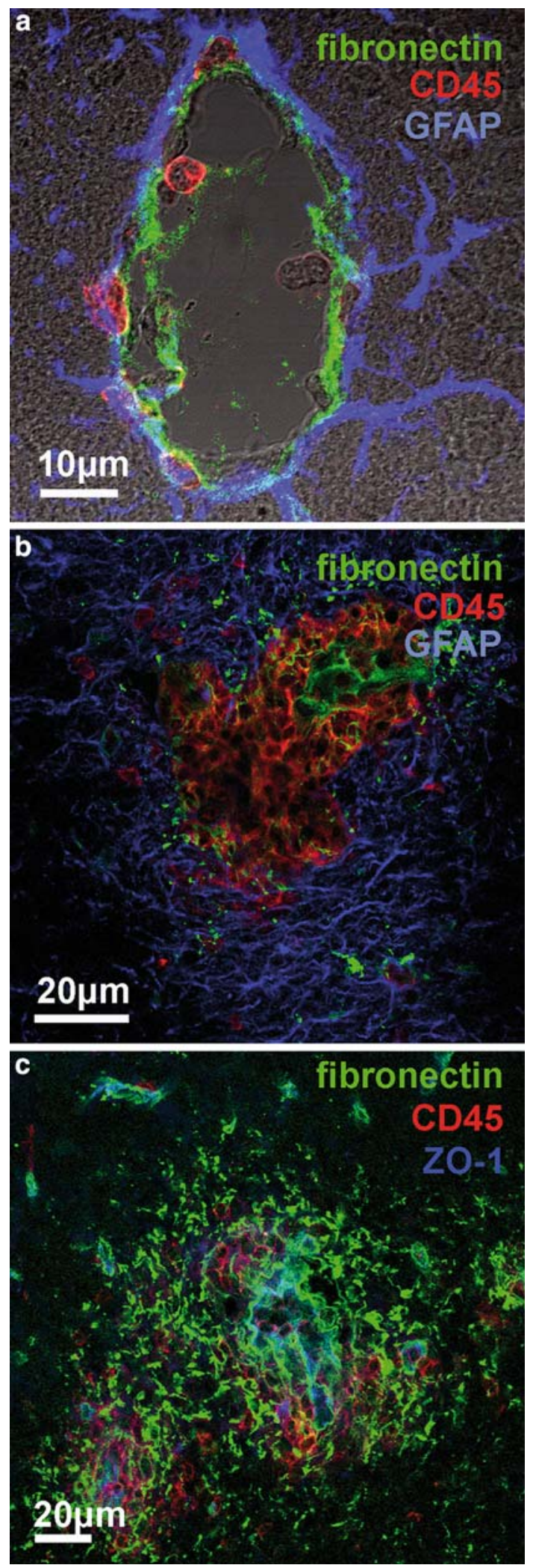

Agrawal et al. [1], we observed a loss of anti- $\beta$-dystroglycan staining in inflamed vessels, where CD45-positive cells were present within the perivascular space (Fig. 2c, d). This
4 Fig. 1 Confocal laser scanning immunohistochemical staining of mouse brains afflicted with EAE, using antibodies against fibronectin, CD45, and GFAP (a, b), and fibronectin, CD45 and ZO-1 (c). a 8 days after immunization, prior to clinical disease. Fibronectin immunoreactivity is restricted to the perivascular basal lamina. CD45-positive cells are concentrated within the perivascular space. b 20 days after immunization, clinical score 1; CD45-positive cells have already penetrated the glial limiting border to get access to the CNS. The fibronectin immunoreactivity has extended beyond the vascular border indicating that fibronectin has leaked from the blood into the CNS. c 16 days after immunization, clinical score 3 , the degree of fibronectin leakage has increased, due to the heavier clinical score, despite shorter time of immunization in comparison with (b)

finding was corroborated at the electron microscopical level by absence of $\beta$-dystroglycan-specific immunogold labeling (data not shown). However, inflamed microvessels devoid of CD45-positive cells were decorated with $\beta$-dystroglycanpositive astroglial endfeet (Fig. 2c, d). Loss of $\beta$-dystroglycan in inflamed postcapillary venules may indicate the role of this member of the dystrophin-dystroglycan complex for the distribution of AQP4 in the astroglial membranes.

Agrin staining is lost in endothelial, not in glial, basal lamina

In contrast to the anti-laminin staining, the anti-agrin immunoreactivity of the endothelial and the glial basal laminae differed. In uninflamed postcapillary venules, both the endothelial and the glial basal lamina were agrinpositive (Fig. 3a). In CNS tissues from mice with a clinical score of 1 , the endothelial anti-agrin staining became weaker, whereas the glial basal lamina maintained in strength of staining (Fig. 3b). Finally, in CNS tissue from animals with severe EAE (clinical score 3), the endothelial agrin was lost and the glial basal lamina agrin was maintained (Fig. 3c). Thus, with increasing severity of CNS inflammation, there is a differential loss of agrin from the endothelial but not from the glial basal lamina within the NVU.

GFAP- and Kir4.1-stainings indicate astroglial hypertrophy

Anti-GFAP staining was used in order to visualize astrocytes in an inflammatory cuff. We found astrocytes enormously hypertrophic around inflamed vessels (Fig. 4a). At places, exuberant astrocytic processes were observed between and around inflammatory cells (Fig. 4b). At the electron microscopic level, GFAP-based intermediate filaments were identified in astrocytic processes by extremely strong immunogold labeling within the inflammatory cuffs (data not shown). This finding confirmed that of 

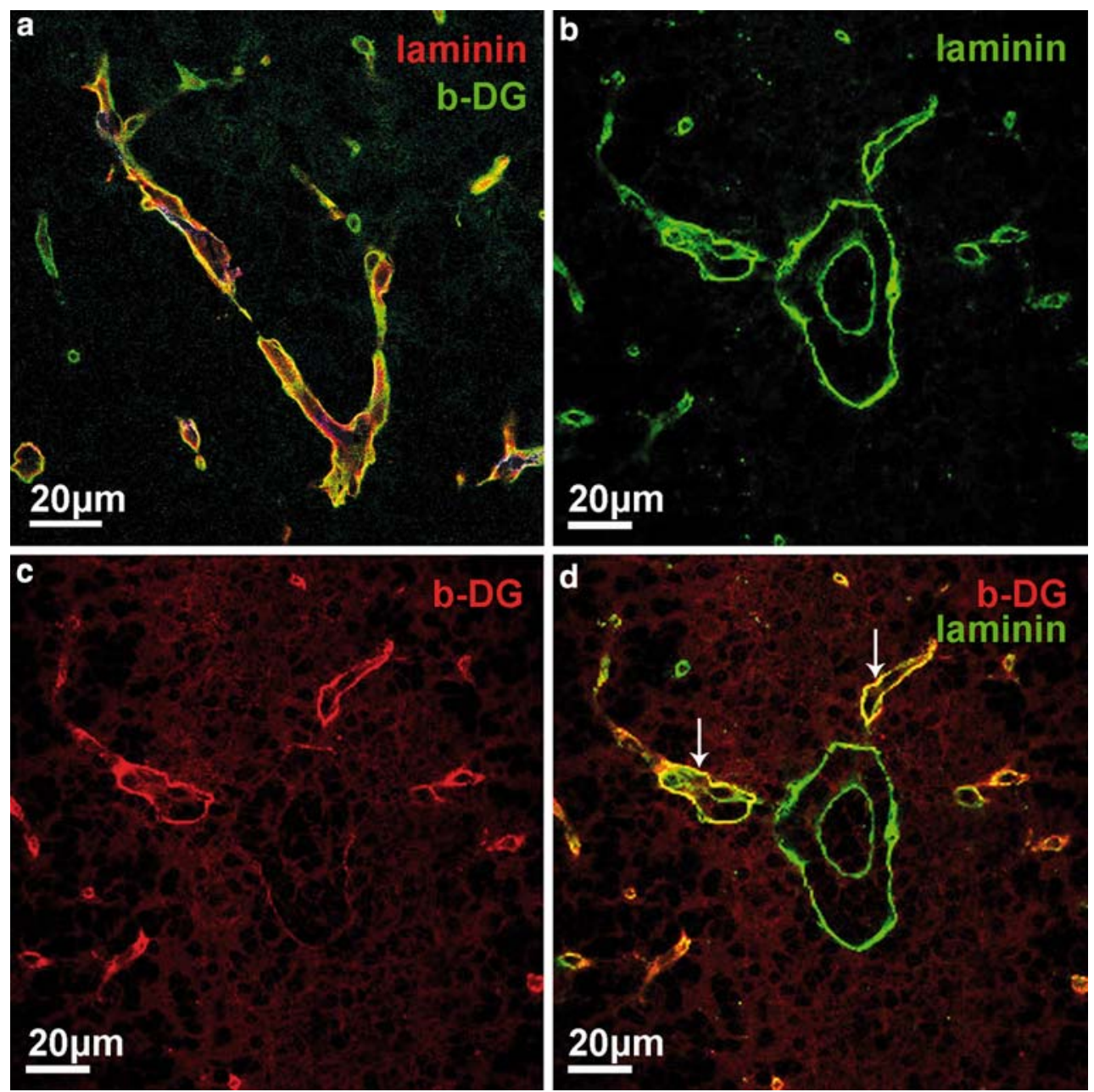

Fig. 2 Confocal laser scanning immunohistochemical staining of mouse brains afflicted with EAE (b-d), using antibodies against $\beta$ dystroglycan and laminin. d Merged image from (b) and (c), b shows separately laminin and (c) $\beta$-dystroglycan. a Normal mouse brain showing a restriction of $\beta$-dystroglycan (green) to the glial endfoot membrane around the laminin-positive subendothelial basal lamina

(red). b-d 14 days after immunization, clinical score 3 . Whereas the capillaries leading to the postcapillary venule (arrows) are immunoreactive for both laminin and $\beta$-dystroglycan, the postcapillary venule in the middle of the picture shows a widened perivascular space with a specific loss of $\beta$-dystroglycan in the glial membrane

Aoki-Yoshino [3] in human autopsies of multiple sclerosis patients. In addition to GFAP, we also used an antibody against the $\mathrm{K}^{+}$channel Kir4.1 in order to characterize perivascular astrocytes within an inflammatory cuff and found a strong anti-Kir4.1 staining in numerous astrocytic perivascular processes (Fig. 4c, d).

AQP4 immunoreactivity seems to be redistributed in inflammatory cuffs

The distribution of AQP4 channels in normal astrocytes is described to be highly polarized towards astrocytic foot processes. Accordingly, in normal brain (Fig. 5a), or in uninflamed vessels of EAE brain (Fig. 5b), the anti-AQP4 staining was clearly restricted to glial endfeet around microvessels. The neuropil between uninflamed vessels

was completely free of any anti-AQP4 staining. However, if comparing EAE tissue obtained from mice without clinical score (Fig. 5b) with EAE tissue obtained from mice with a clinical score 1 (Fig. 5c), and EAE tissue obtained from mice with a clinical score 3 (Fig. 5d), we found-using strictly the identical laser parameters at the confocal laser scanning microscope-a constant increase of anti-AQP4-related fluorescence intensity in the areas of perivascular inflammatory cuffs correlating to the severity of EAE. Western blot analysis of the cerebellum and spinal cords of differently scored EAE animals and healthy controls failed to detect enhanced AQP4 protein levels (data not shown), further supporting the notion that the enhanced AQP4 levels observed by immunofluorescence stainings are restricted to the inflammatory cuffs with the surrounding CNS tissue being unaffected. Due to the 

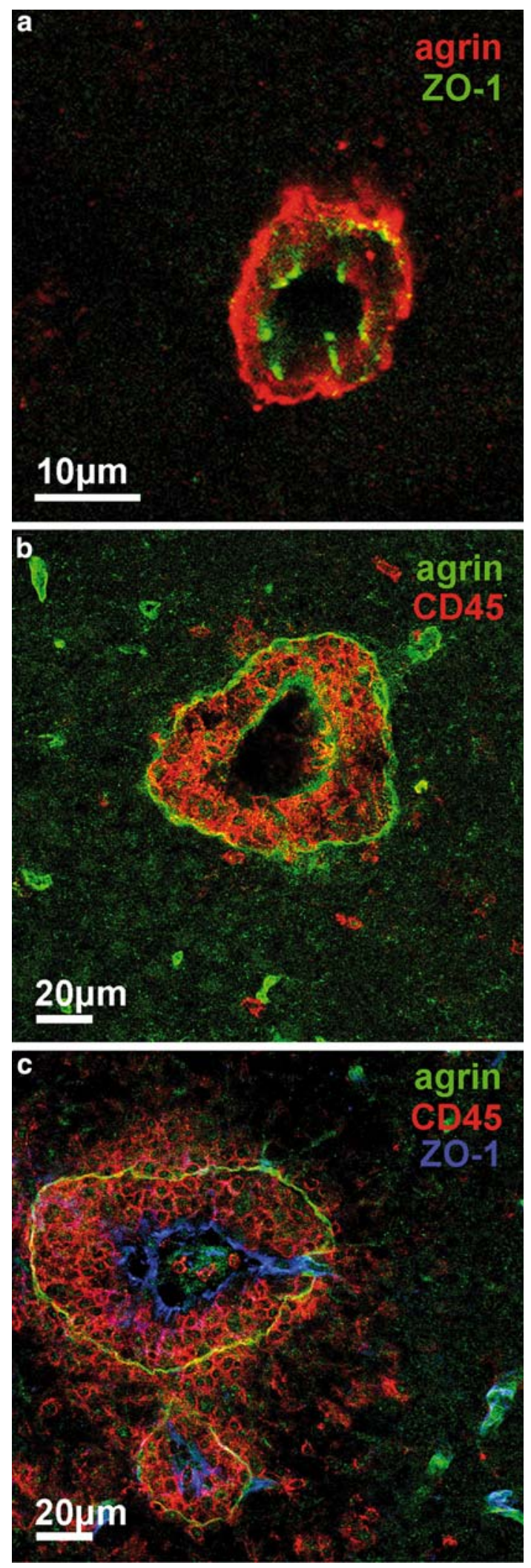

relevance of the question, whether the enhancement of the anti-AQP4 signal in EAE does really reflect a redistribution of AQP4 in astrocytes of an inflammatory cuff, we decided
4 Fig. 3 Confocal laser scanning immunohistochemical staining of mouse brains afflicted with EAE (b, c), using antibodies against agrin and ZO-1 (a), agrin and CD45 (b), and agrin, ZO-1 and CD 45 (c). a Normal mouse brain showing clear restriction of agrin immunoreactivity to the perivascular basal lamina. b 20 days after immunization, clinical score 1. The outer (glial), but not the inner (endothelial) basal lamina is completely intact and maintained concerning agrin content, CD45-positive cells are restricted to the perivascular space of the postcapillary venule. c 12 days after immunization, clinical score 3 . CD45-positive cells have penetrated the perivascular space and invaded the CNS. The inner ring of endothelial basal lamina around the ZO-1-positive endothelial cells is devoid of agrin, whereas the outer ring is still intact and continuous

to perform immunogold labeling and a freeze-fracture analysis of EAE material.

When compared with an uninflamed vessel, which showed AQP4-specific immunogold particles predominantly at the perivascular glial endfoot membranes (Fig. 6a), inflammatory cuffs contained astrocytes revealing immunoreactive gold labeling not only at the perivascular membranes (Fig. 6b, c, small arrows) but also at parenchymal membranes (Fig. 6b, c, large arrows). Figure $6 \mathrm{c}, \mathrm{d}$ shows consecutive sections with immunogold labeling of AQP4 (c) and laminin (d). Membranes covered by a basal lamina were largely occupied by immunogold particles indicating the presence of AQP4 (Fig. 6c, small arrows). The opposing membrane as directed to the neuropil was not covered by a basal lamina but labeled by anti-AQP4-immunogold particles as well (Fig. 6b, c, large arrows) indicating a redistribution of AQP4 over the astrocytic surface. For better clarity, the situation visualized in Fig. 6c, d was drawn in a schematic sketch (Fig. 6e).

Rearrangement of astroglial membranes facing an inflammatory cuff

The freeze-fracture analysis of inflammatory EAE cuffs was extremely difficult due to the lack of the normal morphology of the NVU characterized by astrocytic endfeet, pericytes and endothelial cells. In a cuff, inflammatory cells can be intermingled with neurons or non-astrocytic glial cells, and the astrocytic endfeet are distant from the endothelium, and separated by the perivascular space, which may or may not be filled with inflammatory cells. This may lead to situations, which could not always be accurately interpreted in the freeze-fracture replica. Nevertheless, by investigating numerous replicas of inflammatory cuff-containing cerebellar tissue, we were able to identify a massive alteration of the architecture of astrocytic membranes. Figure 7a shows a blood vessel surrounded by several cells (not seen in the image). The nature of these cells could not be determined; however, they seemed to be inflammatory cells, because in the blood vessel a nucleated blood cell was just leaving the 

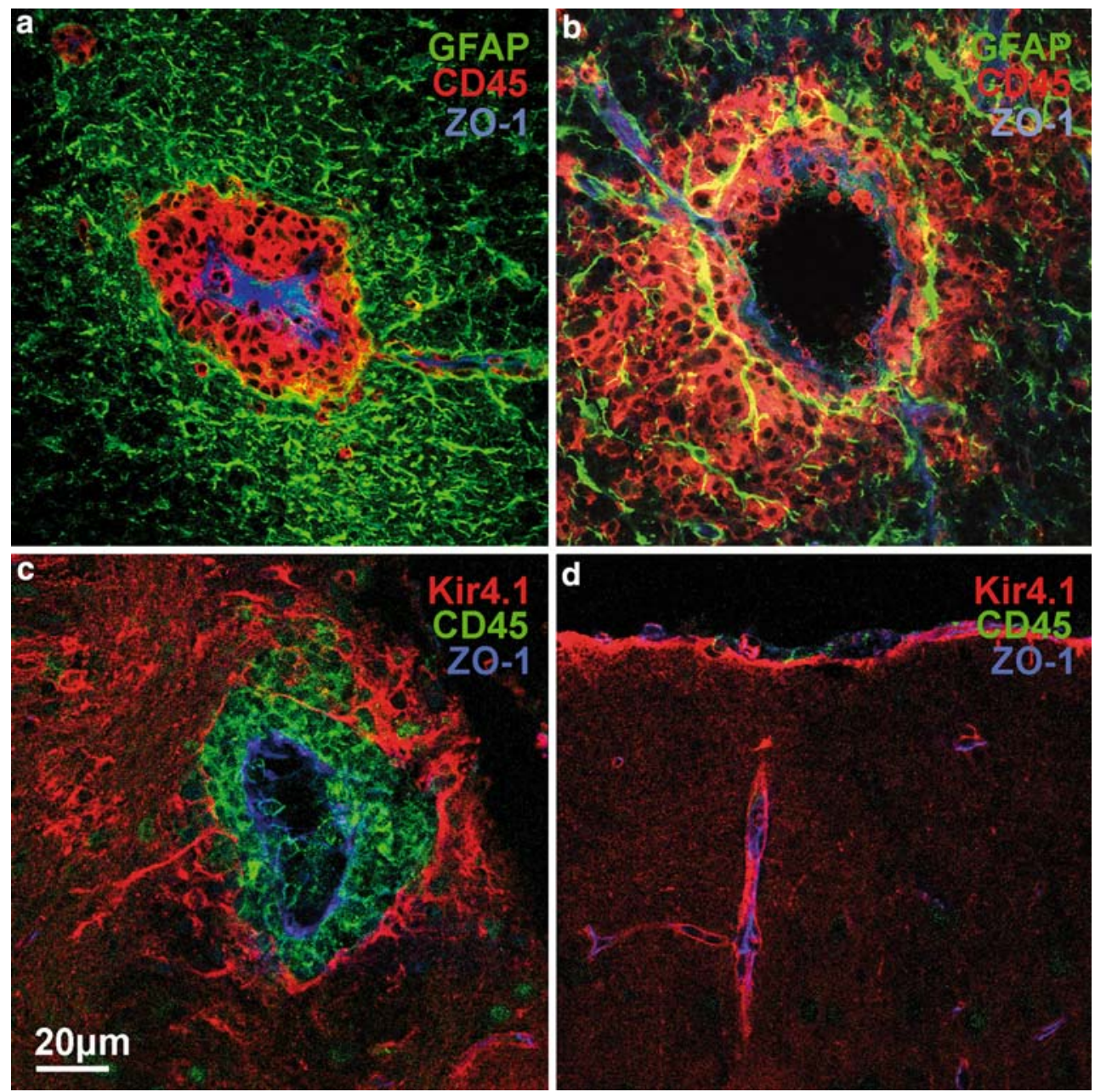

Fig. 4 Confocal laser scanning immunohistochemical staining of mouse brains afflicted with EAE, using antibodies against CD 45, ZO1 and GFAP (a, b) and Kir4.1 (c, d), respectively. a 20 days after immunization, clinical score $1, \mathrm{CD} 45$-positive cells are concentrated within the perivascular space. b 14 days after immunization, clinical score 3, CD45-positive cells have already penetrated the glial limiting

lumen. This could be demonstrated in more detail higher magnified in Fig. $7 \mathrm{~b}$ which showed the opening of the endothelial lining by the extravasating leukocyte coming in contact with the astrocytic endfoot (double-arrow), from which a piece of E-face (AEF in Fig. 7b) can be seen. The cytoplasm of this astrocyte endfoot is seen as an extremely thin layer covered by a cell the E-face of which is seen in Fig. 7c. In our context it is important that the astrocytic Pface irregularly exposed through gaps of this E-face varied considerably in the content of OAPs. Two detailed views (Fig. 7c, d) revealed the extremely different density of OAPs in astrocytic domains not directly contacting the glial limiting membrane.

The astrocytic membrane as shown in Fig. 7d, not only showed few OAPs in the parenchymal membrane, but also border to get access to the CNS. c, d Vessels, 12 days after immunization, clinical score 3. In (d), superficial and perivascular endfeet positive for Kir4.1 are shown. Here, no cuffs are formed, confirming that the alterations seen in (c) are specific for inflammation

an aggregation of intramembraneous particles. Sometimes, it seemed that the particles would form incomplete OAPs. This finding was more clearly visible in another replica of the same tissue, in which an astrocytic parenchymal membrane could be shown not far away from a postcapillary venule (Fig. 8a, b). In this membrane, a large cluster of particles was formed, which was reminiscent of AQP4 lattices, which were found after transfection of cells with the cDNA of the M23 isoform of AQP4 [6].

Another replica analysis from a brain slice of an EAE mouse (clinical score 2) with inflammatory cuffs showed, for the first time in the literature, large lattice-like OAPs in membranes (Fig. 9b, c) not adjacent to basal laminae but to unidentified cells, which nevertheless were non-endothelial and non-glial in nature (asterisk in Fig. 9a). 

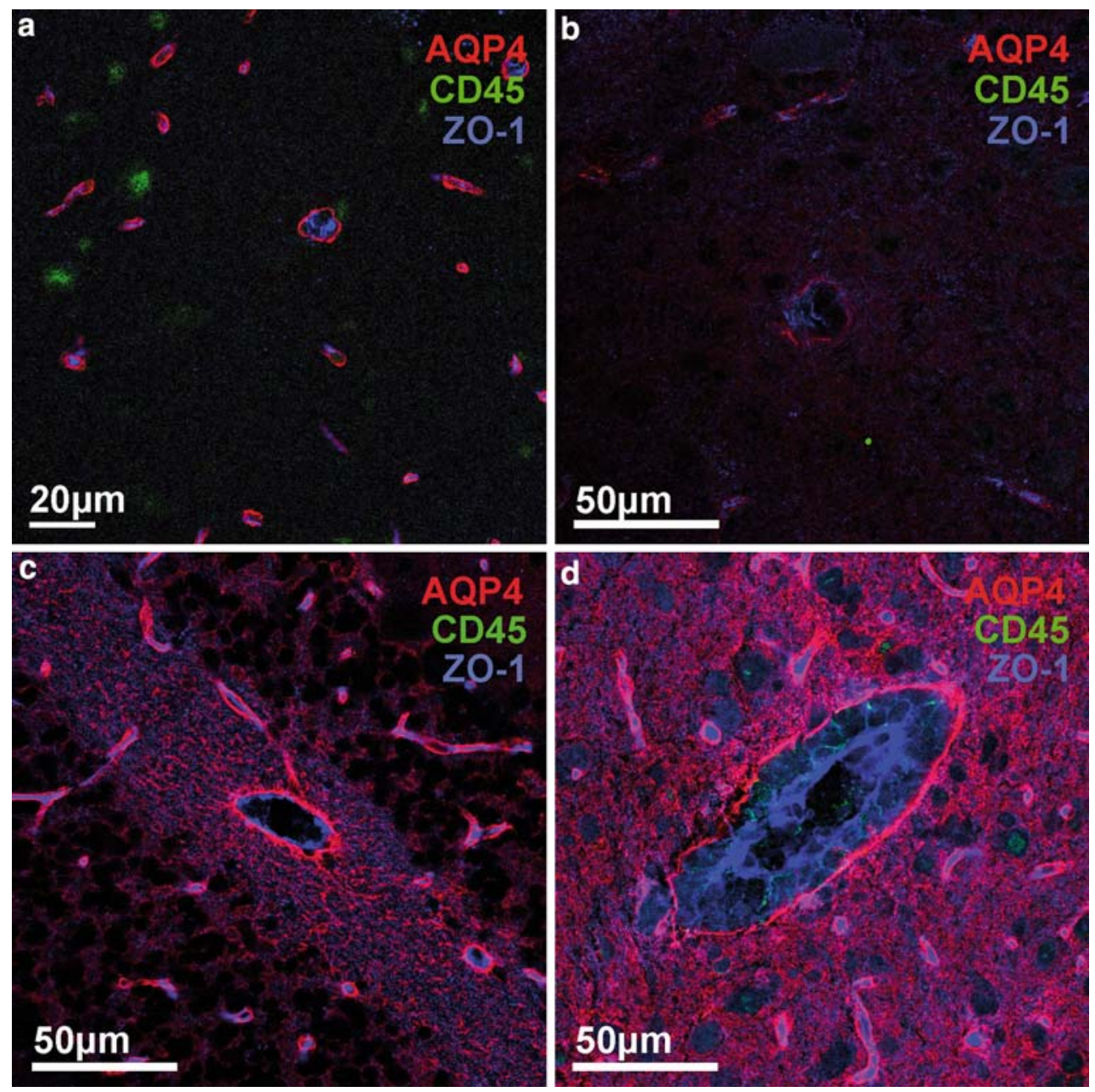

Fig. 5 Confocal laser scanning immunohistochemical staining of mouse brains afflicted with EAE (b-d), using antibodies against AQP4, CD45, and ZO-1. a Normal mouse brain showing no inflammation and a restriction of AQP4 immunoreactivity (red) to the perivascular glial endfeet membranes. In $\mathbf{b}-\mathbf{d}$, the confocal laser scanning parameters were strictly the same in all three images. b 8 days after immunization, without clinical score, only few CD45positive cells have transmigrated the blood-brain barrier. c 20 days

\section{Discussion}

The main finding of this study is the loss of polarized expression of AQP4 on astrocyte endfeet from astrocytes within perivascular inflammatory cuffs during EAE. Within the inflammatory cuff, dystroglycan selectively disappeared from astrocytes and AQP4 redistributed from the endfeet membranes to the parenchymal membranes of the astrocyte body. The degree of both alterations correlated with the amount of CNS inflammation and clinical severity of EAE.

\section{AQP4 in EAE}

AQP4 is the main water channel protein isoform in the CNS and strictly distributed in a polarized fashion.

after immunization, clinical score 1, CD45-positive cells (red) are restricted to the perivascular space, AQP4 immunoreactivity (green) is eventually increased in the neuropil giving raise to the investigation as shown in Figs. 7, 8, and 9. d 11 days after immunization, clinical score 3. CD45-positive cells (green) have now invaded the CNS; in this animal, AQP4 (red) is restricted to the perivascular astroglial endfeet membranes

The astroglial endfoot membranes carry the greatest part of the AQP4 molecules, whereas in non-endfoot membranes AQP4 molecules occur only in low density. Under the conditions of multiple sclerosis, the AQP4 protein is upregulated [3, 28]; under conditions of NMO the AQP4 protein was found to be absent [28]. In order to explain this difference between two clinically related neuroinflammatory diseases, it has to be taken into account that in $\mathrm{NMO}$ the autoantigen is not a myelin component but AQP4 [10]. In the present study, we found not only an apparent increase of the anti-AQP4 immunoreactivity in EAE (Fig. 5) but also a redistribution of AQP4 over the surface of the astrocyte. Both phenomena, increase and redistribution, do not appear to be connected in principle, because in the $\alpha$-syntrophin null mouse the redistribution of AQP4 
Fig. 6 Electron microscopical immunogold labeling using antibodies against AQP4 (a-c) and laminin (d). a Normal mouse brain showing restriction of AQP4-specific gold grains to the perivascular astroglial endfoot membrane (arrows). b 15 days after immunization, clinical score 2, AQP4-specific gold grains are no more restricted to astroglial endfeet membranes (small arrows point to endfoot staining, large arrows to parenchymal staining). c, d 15 days after immunization, clinical score 2 , consecutive sections stained against AQP4 (c) and laminin (d). AQP4-specific gold grains in (c) are no more restricted to astroglial endfeet membranes (small arrows point to endfoot staining, large arrows to parenchymal staining). e Schematic reconstruction of (c) and (d) for better understanding of the topological situation as shown in $\mathbf{c}, \mathbf{d}$. The anti-AQP4-gold labeling of the astroglial parenchymal membrane indicates the redistribution of AQP4 over the surface of the glial cell. A Astrocyte, $M$ monocyte in the perivascular space
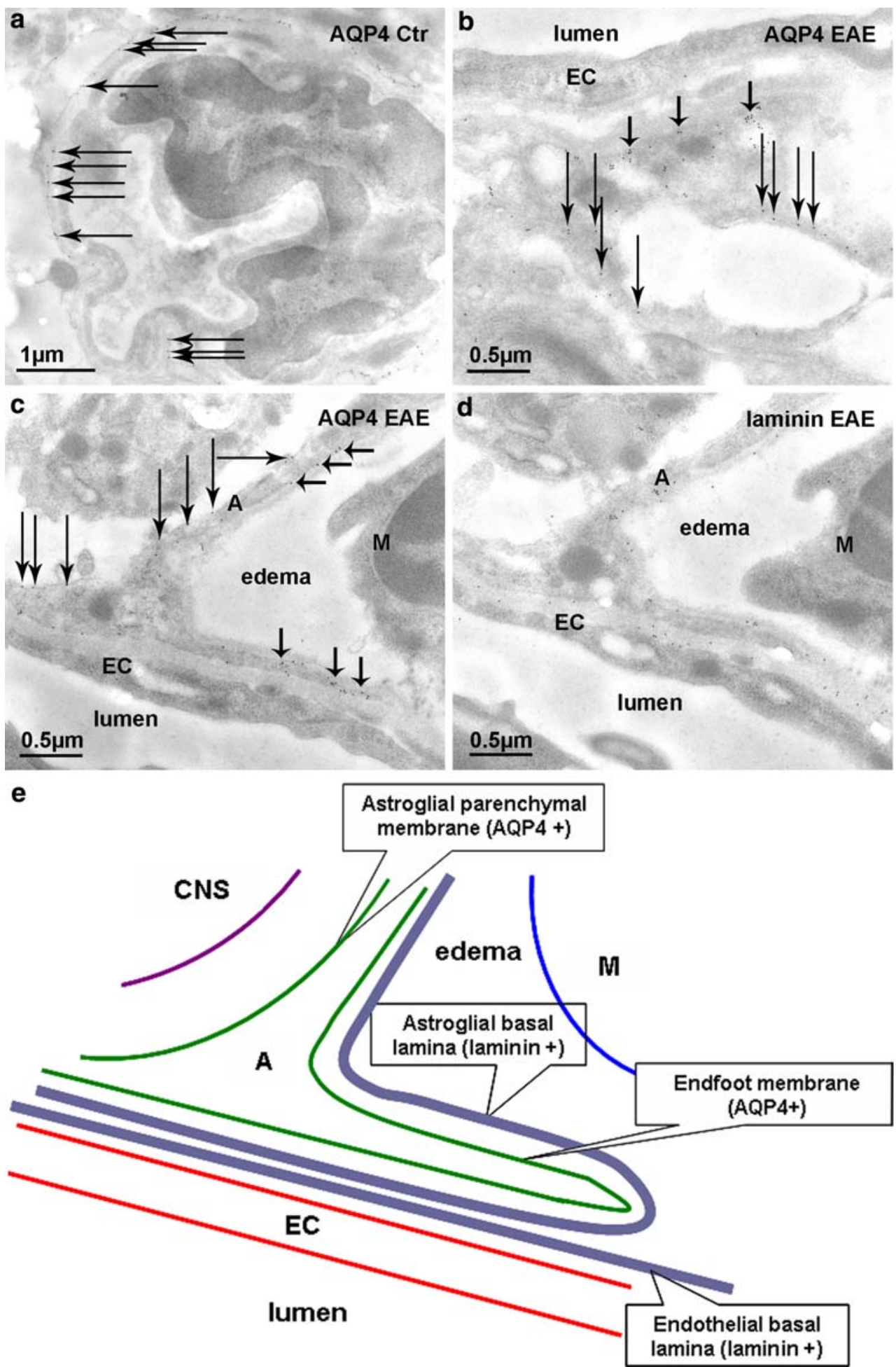

was not associated with an alteration of AQP4 expression [17]. The comparison of the $\alpha$-syntrophin null mouse with the EAE shows that the stability of the AQP4 molecule in the endfoot membrane not only depends on the presence of $\alpha$-syntrophin, but also on the integrity of complex molecular interactions at the glio-vascular interface, which may be disturbed in EAE. Among the molecules, which are essential for the integrity of the BBB in general and the protection of the brain against leukocyte diapedesis in particular, is dystroglycan. 

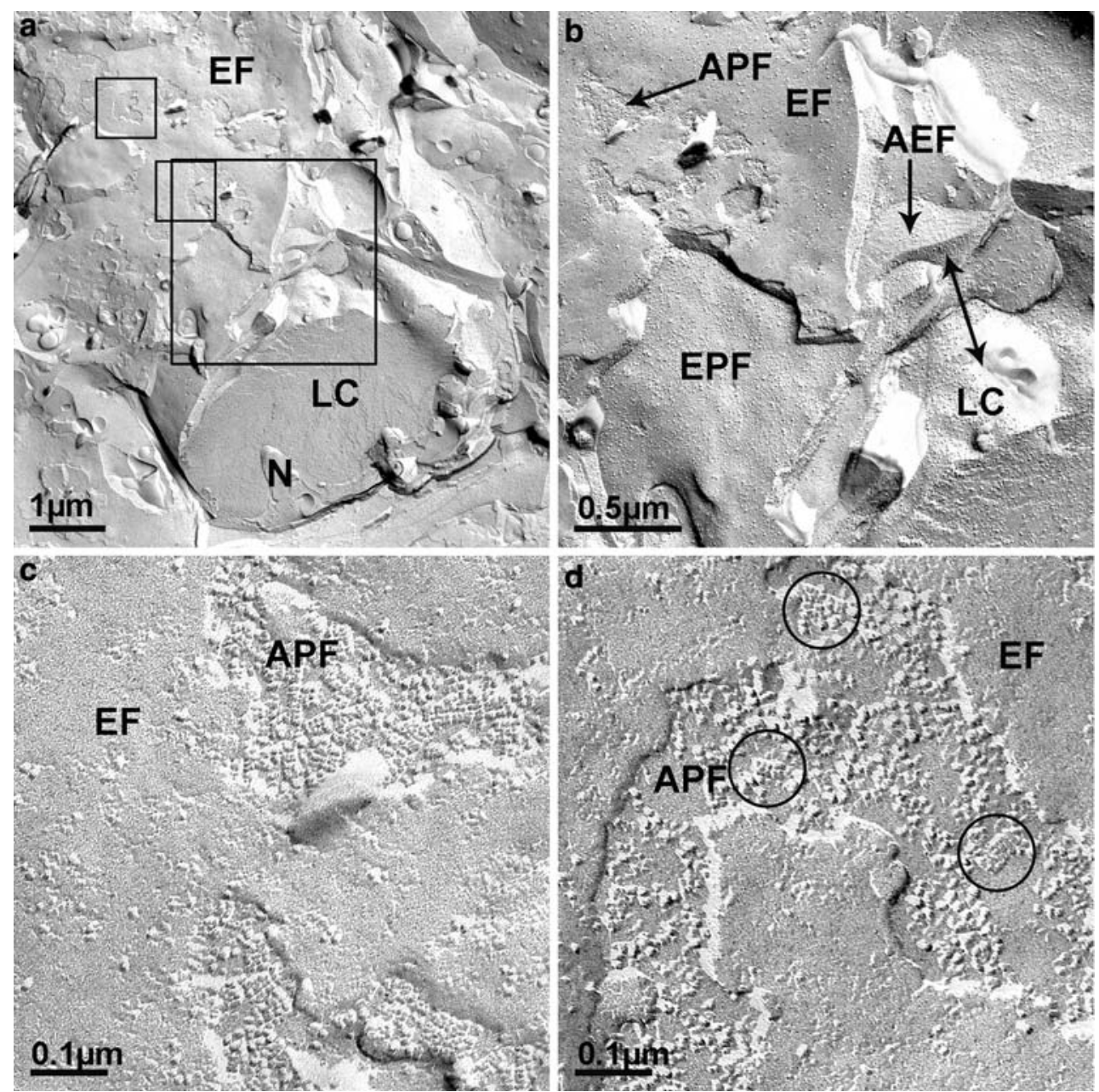

Fig. 7 Freeze-fracture replica of a vibratome section from a mouse cerebellum, 14 days after immunization, clinical score 2. The presence of cuffs was shown by light microscopy. a Low magnified overview of a vessel, filled with a leukocyte $(L C)$. $N$ Nucleus of the leukocyte. The large box frames the area shown in (b) at higher magnification. $A E F$ E-face of an astrocytic endfoot occupied by densely packed OAP pits (difficult to recognize at this magnification).

\section{Dystroglycan}

Cleavage of astrocytic dystroglycan by matrix metalloproteinases 2 and 9 (MMP-2, -9) at sites of leukocyte diapedesis across the BBB in EAE has first been reported by Agrawal et al. [1], and disappearance of anti-dystroglycan immunoreactivity has been confirmed by the present study (Fig. 2). Dystroglycan and the DDC have been described in the CNS, including synapses, astrocytes and endothelial cells $[5,31,40]$. In astrocytes, actin filaments of the cytoskeleton are either linked to dystrophin or utrophin, which is also called dystrophin-related protein. Dystrophin is connected via its amino-terminal domain to dystroglycan, which consists of an $\alpha$ - and a $\beta$-subunit. Both subunits are encoded by a single gene and are formed by cleavage of a precursor protein into two mature proteins
The double-arrow marks the direct access of the leukocyte to the subendfoot space. $E P F$ E-face of the lumenal endothelial cell membrane. $A P F$ P-face of the astrocyte directed away from the vessel. $E F$ E-face of an unidentified cell directly adjacent to the astrocyte. c, $\mathbf{d}$ Higher magnifications of the replica site framed with the small boxes in $\mathbf{a}$. In c, the P-face of the astrocyte $(A P F)$ contains many OAPs, whereas at a site (d) distant from site (c), only few OAPs are present (circled)

that form a tight non-covalent complex [7, 35]. The transmembrane protein $\beta$-dystroglycan anchors $\alpha$-dystroglycan to the cell membrane and the cytoskeleton via its linkage to the $\mathrm{C}$-terminal domain of dystrophin. The brainselective deletion of the dystroglycan gene was described to cause brain malformations such as disorganization of cortical layering and aberrant migration of granule cells [14]. On the other hand, absence of dystroglycan by means of MMP-dependent degradation seems to be a prerequisite for entry of inflammatory cells into the CNS parenchyma during EAE and establishment of clinical disease [1]. Indeed, in the present study absence of any dystroglycan immunoreactivity seemed to correlate strictly to the presence of CD45-positive inflammatory cells: after leaving the EAE plaque, the blood vessel lining was again decorated by anti-dystrogycan immunoreactivity. 


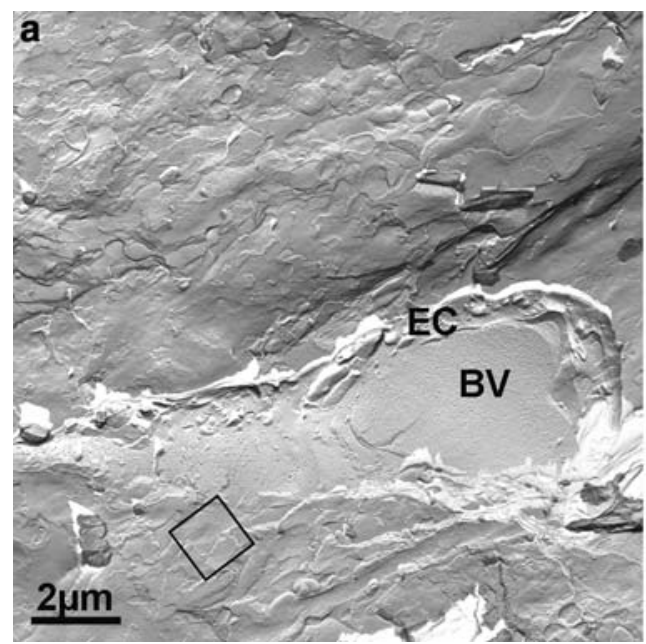

Fig. 8 Freeze-fracture replica of a vibratome section from a mouse cerebellum, 14 days after immunization, clinical score 2. The presence of cuffs was shown by light microscopy. a Low magnified overview of a blood vessel $(B V)$ with endothelial cells $(E C)$. The box frames the site which is shown in (b) at higher magnification. b Detail of the astrocyte which is perivascular the blood vessel as shown in (a).

\section{AQP4/OAP and agrin}

Another novel observation, namely the detection of AQP4 as well as OAPs away from the astroglial endfoot membrane in such an inflammatory cuff has interesting implications. In the healthy brain tissue, AQP4 immunoreactivity and OAPs were restricted to the astroglial endfoot membrane adjacent to CNS microvessels, clearly demonstrating the polarization of astrocytes in dependence of the presence of components of the basal lamina. Among these components, the heparan sulfate proteoglycan agrin has recently been identified as a candidate molecule for inducing astrocyte polarity, because the endothelial agrin isoform A0B0 was able to cluster OAPs in the astroglial membrane [19]. Previous findings have shown that in human glioblastoma (1) agrin was lost where the bloodbrain barrier tight junctions were disturbed [21], (2) AQP4 was upregulated [25] and redistributed [33], and (3) OAPs disappeared [18]. These findings together tempted us to speculate that under pathological conditions AQP4 can also occur as non-OAPs (increase of AQP4 protein amount and decrease of OAPs did not allow another conclusion) and that agrin would be responsible to cluster AQP4 tetramers as a supramolecular complex visible in the freeze-fracture replica.

AQP4 exists in six different splice variants [13] known as the AQP4 a-f isoforms. AQP4a is identical with the previously named $\mathrm{M} 1$ isoform, and $\mathrm{AQP} 4 \mathrm{c}$ is identical with the previously named M23 isoform. M1 is 22 amino acids longer at the N-terminus than M23 [8]. Transfection of AQP4-M23 but not of AQP4-M1 into CHO cells induces

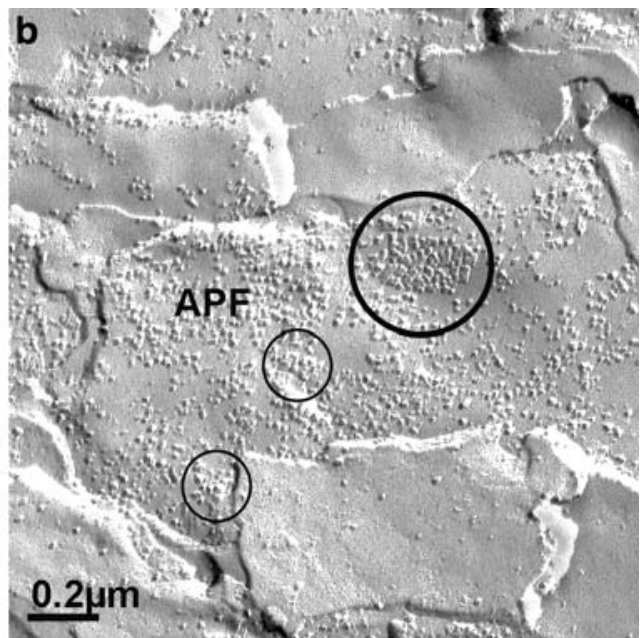

$A P F$ P-face of the astrocyte occupied by many intramembranous particles few of them are OAPs (marked by small circles). The complex labeled by the large circle is reminiscent of OAP-based lattices known to be formed after transfection with M23-AQP4 (see text)

the formation of OAPs in the transfected cells [6]. If both AQP4 isoforms were cotransfected, the OAPs formed in the transfectants closely resembled those found on astrocytic endfeet in vivo. In parallel, the water permeability coefficient of the M23 water channel if transfected into LLC-PK 1 cells was much greater than that of the M1 water channel [26].

Recently, we demonstrated that the endothelial agrin isoform A0B0 did not change the expression level of $\mathrm{M} 1$ and M23, but that the neuronal agrin isoform A4B8 was able to upregulate M23 and downregulate M1, thus increasing the M23/M1 ratio by the factor of about 6 [19]. However, this change of the ratio was not reflected by a considerable change of the OAP density in the astrocytic membrane. The OAP density was only changed if astrocytes were under the influence of the endothelial agrin A0B0. In the agrin-deficient mouse, the amount of OAPs was drastically decreased without change in the immunohistochemical stainings using antibodies against AQP4 [20]. This is additional evidence arguing that indeed agrin is essentially responsible for the aggregation of AQP4 subunits to OAPs.

\section{AQP4/OAP and dystroglycan in EAE}

Although we could reproduce the finding of Agrawal et al. [1] that in focal EAE inflammatory cuffs, immunostaining for dystroglycan is lost we cannot fully confirm their previous finding that presence of agrin staining remained unchanged. Our present study revealed a decrease of antiagrin-immunofluorescence staining in the endothelial, but 


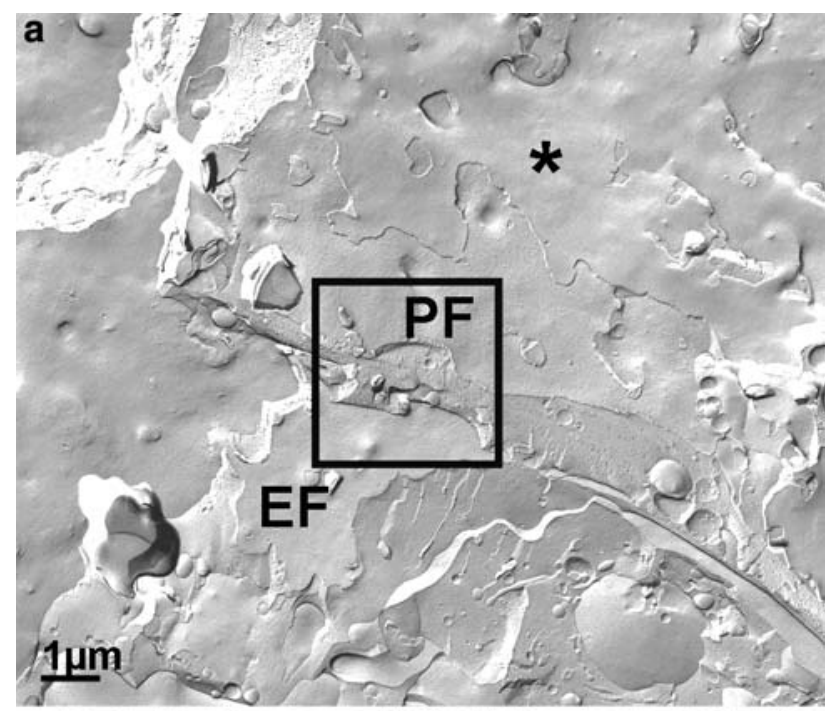

b
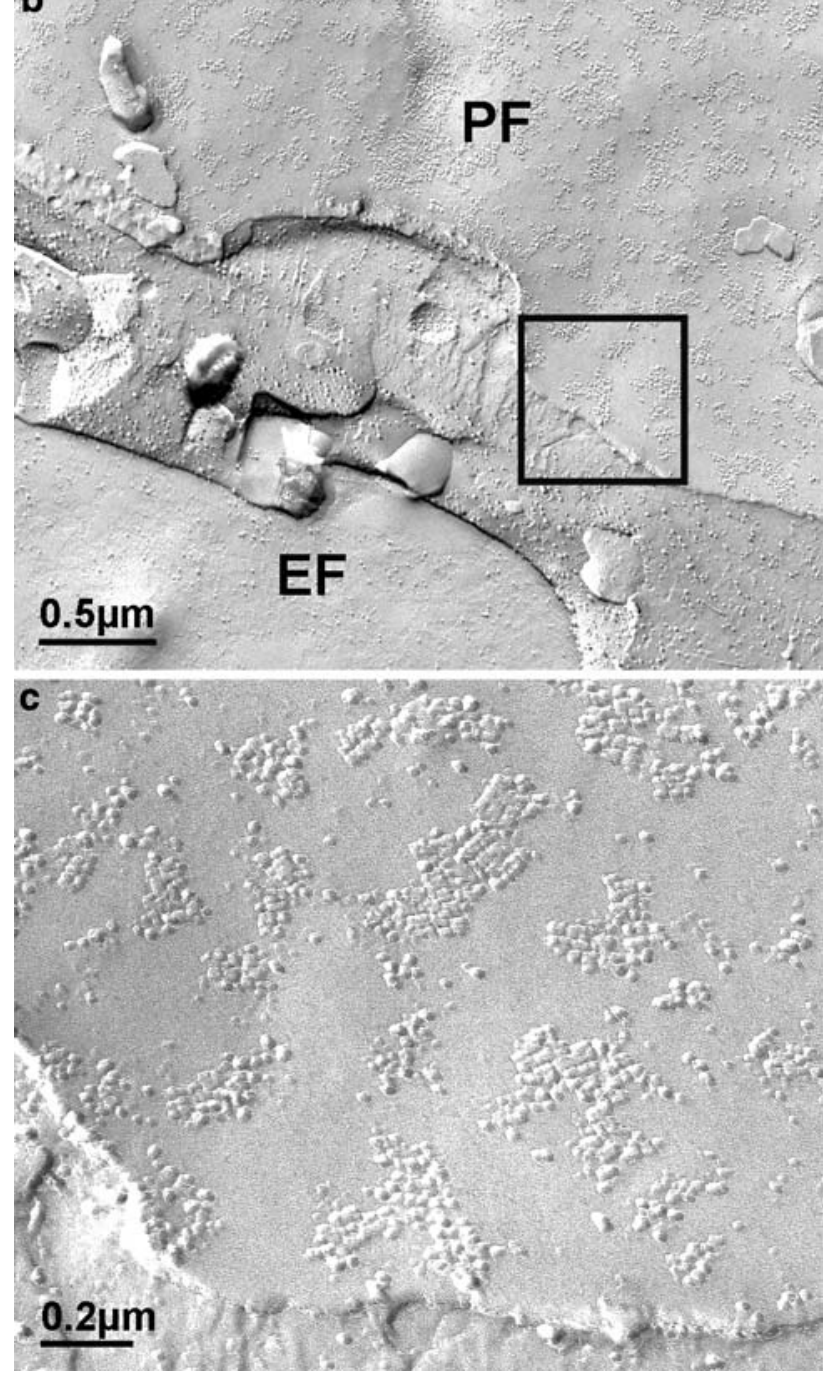

not in the glial basal lamina. It will remain to be shown whether this is due to different pathomechanisms applying to clinical EAE in SJL mice (this study) versus C57BL/6
Fig. 9 Freeze-fracture replica of a vibratome section from an EAEcuff, 14 days after immunization, clinical score 2. b Corresponds to the area framed in (a) at a higher magnification, $\mathbf{c}$ detail of the framed area in (b). An astrocytic process is in contact with a non-endothelial cell and non-glial cell, thus presumably an inflammatory monocytic cell (asterisk in a). In (b), this process is shown in more detail: $P F$ P-face, $E F$ E-face which contains many OAP pits (not visible at this magnification). The area between $\mathrm{P}$ - and E-face corresponds to the astrocytic cytoplasm. c High-power magnification of the P-face in (b) showing large OAP lattices normally not seen in astrocytes in vivo

mice [1]. In any case, agrin in the glial basal lamina anchoring the astrocytic endfeet remained stable in the inflamed vessels, whereas dystroglycan staining disappeared specifically in the astrocytic endfoot domains. In freeze-fracture replicas, we found an enlarged amount of OAPs in astrocytes, not only in vessel-associated domains, but also in parenchymal domains apart from the contact with vessels as well. This implied that in the presence of agrin, but absence of dystroglycan, OAPs can be formed but their targeting may not be restricted to the endfoot domain.

This finding has an important consequence: in former studies we repeatedly stressed the point that if agrin influences the clustering of OAPs, dystroglycan should be a member of the OAP complex, because agrin does not bind to AQP4 directly but rather to $\alpha$-dystroglycan. However, here we observed that under conditions of CNS inflammation, dystroglycan but not OAPs disappeared ([1], present study, Fig. 2). On the contrary, the density of OAPs in parenchymal membranes of the astrocytes seemingly increased, and in some areas, the OAPs grew to lattices reminiscent of those, which were induced after transfection of M23 AQP4 [6]. This was in particular the case, where the astrocyte was in direct contact to cells which were though unidentified neither endothelial nor glial cells assuming that they could be inflammatory cells. This point has to be verified in separate in vitro studies, which are currently designed. Taken together, we conclude that dystroglycan directs the correct location of the OAPs in endfoot domains of astroglial cells, whereas agrin is involved in the aggregation of AQP4 to OAPs.

The redistribution of OAPs in inflamed vessels under the conditions of dystroglycan degradation should also have functional consequences for the water flux in the inflamed brain. It has been well known for many years that there is a colocalization of the weakly inwardly rectifying potassium channel Kir4.1 and AQP4 at the membrane of astrocytic endfeet surrounding CNS microvessels [16]. Both channels are essentially involved in the spatial buffering of the extracellular $\mathrm{K}^{+}$concentration: neuronally (synaptically) induced increase of extracellular $\left[\mathrm{K}^{+}\right]$is answered by $\mathrm{K}^{+}$ influx, and, osmotically driven, water influx into the glial cell, which is followed by a distant efflux where the density 
of potassium and water channels is highest, namely at the endfoot membrane. Thus, water fluxes are driven by ion fluxes, and $\mathrm{K}^{+}$-clearance is coupled to water flux [27]. Accordingly, in the $\alpha$-syntrophin-deficient mouse, in which AQP4 is dislocalized across the glial surface, the $\mathrm{K}^{+}$clearance was delayed [2] indicating that $\mathrm{K}^{+}$uptake would be facilitated, if accompanied by water flux and delayed, if water transporters were dislocalized. In human glioblastoma tissue we were able to observe a redistribution of both anti-AQP4- and anti-Kir4.1-immunoreactivities over the surface of the glioma cells [34]. Considering inflammation in EAE, the disturbance in OAP distribution as a result of dystroglycan degradation could lead to a water flux, which would increase the vasogenic edema. Vasogenic edema rises as a consequence of blood-brain barrier disruption (from the blood compartment), and by water influx after $\mathrm{K}^{+}$-clearance and misdirection of water not only to the astrocytic endfoot membranes but also to the lateral domains associated with the interstitial space (from the neural compartment). Further experiments are needed to understand the molecular networks at the glio-vascular interface, which are disorganized under the conditions of EAE and possibly multiple sclerosis, which contribute to edema formation.

Acknowledgments The expert technical assistance of Therese Périnat is acknowledged. We thank Ria Knittel for skilful help with freezefracturing and Gabi Frommer-Kästle with ultrathin sectioning. This study was funded by the Hertie Foundation Germany (grant 1.01.1/07/ 003) to HW and BE and by the Theodore Ott Foundation to BE.

\section{References}

1. Agrawal S, Anderson P, Durbeej M, Van Rooijen N, Ivars F, Opdenakker G, Sorokin LM (2006) Dystroglycan is selectively cleaved at the parenchymal basement membrane at sites of leukocyte extravasation in experimental autoimmune encephalomyelitis. J Exp Med 203:1007-1019

2. Amiry-Moghaddam M, Otsuka T, Hurn PD, Traystman RJ, Haug F-M, Froehner SC, Adams ME, Neely JD, Agre P, Ottersen OP, Bhardwaj A (2003) An $\alpha$-syntrophin-dependent pool of AQP4 in astroglial end-feet confers bidirectional water flow between blood and brain. Proc Natl Acad Sci USA 100:2106-2111

3. Aoki-Yoshino K, Uchihara T, Duyckaerts C, Nakamura A, Hauw J-J, Wakayama Y (2005) Enhanced expression of aquaporin 4 in human brain with inflammatory diseases. Acta Neuropathol 110:281-288

4. Barber AJ, Lieth E (1997) Agrin accumulates in the brain microvascular basal lamina during development of the bloodbrain barrier. Dev Dyn 208:62-74

5. Calogero A, Pavoni E, Gramaglia T, D'Amati G, Ragona G, Brancaccio A, Petrucci TC (2006) Altered expression of $\alpha$-dystroglycan subunit in human gliomas. Cancer Biol Therapy 5:441448

6. Furman CS, Gorelick-Feldman DA, Davidson KGV, Yasumura T, Neely JD, Agre P, Rash JE (2003) Aquaporin-4 square array assembly: opposing actions of M1 and M23 isoforms. Proc Natl Acad Sci USA 100:13609-13614
7. Holt KH, Crosbie RH, Venzke DP, Campbell KP (2000) Biosynthesis of dystroglycan: processing of a precursor propeptide. FEBS Lett 468:79-83

8. Jung JS, Bhat RV, Preston GM, Guggino WB, Baraban JM, Agre $P$ (1994) Molecular characterization of an aquaporin cDNA from brain: candidate osmoreceptor and regulator of water balance. Proc Natl Acad Sci USA 91:13052-13056

9. Kniesel U, Wolburg H (2000) Tight junctions of the blood-brain barrier. Cell Mol Neurobiol 20:57-76

10. Lennon VA, Kryzer TJ, Pittock SJ, Verkman AS, Hinson SR (2005) IgG marker of optic-spinal multiple sclerosis binds to the aquaporin-4 water channel. J Exp Med 202:473-477

11. Liebner S, Fischmann A, Rascher G, Duffner F, Grote E-H, Kalbacher H, Wolburg H (2000) Claudin-1 and claudin-5 expression and tight junction morphology are altered in blood vessels of human glioblastoma multiforme. Acta Neuropathol 100:323-331

12. Lindahl P, Johansson BR, Levéen P, Betsholtz C (1997) Pericyte loss and microaneurysm formation in PDGF-B-deficient mice. Science 277:242-245

13. Moe SE, Sorbo JG, Sogaard R, Zeuthen T, Ottersen OP, Holen T (2008) New isoforms of rat aquaporin-4. Genomics 91:367377

14. Moore SA, Saito F, Chen J, Michele DE, Henry MD, Messing A, Cohn RD, Ross-Barta SE, Westra S, Williamson RA, Hoshi T, Campbell KP (2002) Deletion of brain dystroglycan recapitulates aspects of congenital muscular dystrophy. Nature 418:422-425

15. Nag S (1996) Cold-injury of the cerebral cortex: immunolocalization of cellular proteins and blood-brain barrier permeability studies. J Neuropathol Exp Neurol 55:880-888

16. Nagelhus EA, Mathisen TM, Ottersen OP (2004) Aquaporin-4 in the central nervous system: cellular and subcellular distribution and coexpression with Kir4.1. Neuroscience 129:905-913

17. Neely JD, Amiry-Moghaddam M, Ottersen OP, Froehner SC, Agre P, Adams ME (2001) Syntrophin-dependent expression and localization of aquaporin-4 water channel protein. Proc Natl Acad Sci USA 98:14108-14113

18. Neuhaus J (1990) Orthogonal arrays of particles in astroglial cells: quantitative analysis of their density, size, and correlation with intramembranous particles. Glia 3:241-251

19. Noell S, Fallier-Becker P, Beyer C, Kröger S, Mack AF, Wolburg $\mathrm{H}$ (2007) Effects of agrin on the expression and distribution of the water channel protein aquaporin-4 and volume regulation in cultured astrocytes. Eur J Neurosci 26:2109-2118

20. Noell S, Fallier-Becker P, Deutsch U, Mack AF, Wolburg H (2009) Agrin defines polarized distribution of orthogonal arrays of particles in astrocytes. Cell Tissue Res (in press)

21. Rascher G, Fischmann A, Kröger S, Duffner F, Grote E-H, Wolburg H (2002) Extracellular matrix and the blood-brain barrier in glioblastoma multiforme: spatial segregation of tenascin and agrin. Acta Neuropathol 104:85-91

22. Rash JE, Davidson KGV, Yasumura T, Furman CS (2004) Freeze-fracture and immunogold analysis of aquaporin-4 (AQP4) square arrays, with models of AQP4 lattice assembly. Neuroscience 129:915-934

23. Rash JE, Yasumura T, Hudson CS, Agre P, Nielsen S (1998) Direct immunogold labeling of aquaporin-4 in square arrays of astrocyte and ependymocyte plasma membranes in rat brain and spinal cord. Proc Natl Acad Sci USA 95:11981-11986

24. Rosso C, Remy P, Creange A, Brugieres P, Cesaro P, Hosseini H (2006) Diffusion-weighted MR imaging characteristics of an acute strokelike form of multiple sclerosis. Am J Neuroradiol 27:1006-1008

25. Saadoun S, Papadopoulos MC, Davies DC, Krishna S, Bell BA (2002) Aquaporin-4 expression is increased in oedematous human brain tumours. J Neurol Neurosurg Psychiatry 72:262-265 
26. Silberstein C, Bouley R, Huang Y, Fang P, Pastor-Soler N, Brown D, Van Hoek AN (2004) Membrane organization and function of M1 and M23 isoforms of aquaporin-4 in epithelial cells. Am J Physiol 287:F501-F511

27. Simard M, Nedergaard M (2004) The neurobiology of glia in the context of water and ion homeostasis. Neuroscience 129:877896

28. Sinclair C, Kirk J, Herron B, Fitzgerald U, McQuaid S (2007) Absence of aquaporin-4 expression in lesions of neuromyelitis optica but increased expression in multiple sclerosis lesions and normal-appearing white matter. Acta Neuropathol 113:187-194

29. Sixt M, Engelhardt B, Pausch F, Hallmann R, Wendler O, Sorokin LM (2001) Endothelial cell laminin isoforms, laminins 8 and 10, play decisive roles in T cell recruitment across the bloodbrain barrier in experimental autoimmune encephalomyelitis. J Cell Biol 153:933-946

30. Stamatovic SM, Dimitrijevic OB, Keep RF, Andjelkovic AV (2006) Inflammation and brain edema: new insights into the role of chemokines and their receptors. Acta Neurochir Suppl 96:444450

31. Tian M, Jacobsen C, Gee SH, Campbell KP, Carbonetto S, Jucker M (1996) Dystroglycan in the cerebellum is a laminin $\alpha 2$ chain binding protein at the glial-vascular interface and is expressed in Purkinje cells. Eur J Neurosci 8:2739-2747

32. Uboldi C, Doring A, Alt C, Estess P, Siegelman M, Engelhardt B (2008) L-Selectin-deficient SJL and C57BL/6 mice are not resistant to experimental autoimmune encephalomyelitis. Eur J Immunol 38:2156-2167
33. Warth A, Kröger S, Wolburg H (2004) Redistribution of aquaporin-4 in human glioblastoma correlates with loss of agrin immunoreactivity from brain capillary basal laminae. Acta Neuropathol 107:311-318

34. Warth A, Mittelbronn M, Wolburg H (2005) Redistribution of the water channel protein aquaporin- 4 and the $\mathrm{K}^{+}$channel protein Kir4.1 differs in low- and high-grade human brain tumors. Acta Neuropathol 109:418-426

35. Winder SJ (2001) The complexities of dystroglycan. Trends Biochem Sci 26:118-124

36. Wolburg H (1995) Orthogonal arrays of intramembranous particles. A review with special reference to astrocytes. J Brain Res 36:239-258

37. Wolburg H, Lippoldt A, Ebnet K (2006) Tight junctions and the blood-brain barrier. In: Gonzales Mariscal L (ed) Tight junctions. Landes Bioscience, Georgetown, pp 175-195

38. Wolburg H, Wolburg-Buchholz K, Kraus J, Rascher-Eggstein G, Liebner S, Hamm S, Duffner F, Grote E-H, Risau W, Engelhardt B (2003) Localization of claudin-3 in tight junctions of the blood-brain barrier is selectively lost during experimental autoimmune encephalomyelitis and human glioblastoma multiforme. Acta Neuropathol 105:586-592

39. Yeung D, Manias JL, Stewart DJ, Nag S (2008) Decreased junctional adhesion molecule-A expression during blood-brain barrier breakdown. Acta Neuropathol 115:635-642

40. Zaccaria ML, Di Tommaso F, Brancaccio A, Paggi P, Petrucci TC (2001) Dystroglycan distribution in adult mouse brain: a light and electron microscopy study. Neuroscience 104:311-324 